\title{
New capability for indirect neutron capture measure- ments: The DICER instrument at LANSCE
}

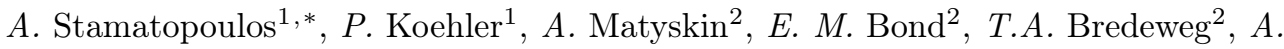 \\ Couture $^{1}$, B. Di Giovine ${ }^{1}$, M.E. Fassbender ${ }^{2}$, A. C. Hayes-Sterbenz ${ }^{3}$, A. L. Keksis ${ }^{2}$, \\ $K$. Parsons ${ }^{4}, G$. Rusev ${ }^{2}, J$. Ullmann ${ }^{1}$, and $C$. Vermeulen ${ }^{2}$ \\ ${ }^{1}$ Physics division, Los Alamos National Laboratory, 87545, NM, USA \\ ${ }^{2}$ Chemistry Division, Los Alamos National Laboratory, 87545, NM, USA \\ ${ }^{3}$ Theoretical Division, Los Alamos National Laboratory, 87545, NM, USA \\ ${ }^{4}$ X-Computational Physics Division, Los Alamos National Laboratory, 87545, NM, USA
}

\begin{abstract}
The Device for Indirect Capture on Radionuclides (DICER) implements a new indirect technique for $(n, \gamma)$ studies in which the neutron capture rate is determined from analysis of resonance neutron transmission data. The DICER instrument and associated radionuclide production at the Isotope Production Facility (IPF), both at the Los Alamos Neutron Science Center (LANSCE), as well radioactive sample fabrication, have been under development in the last few years. First measurements on a radioactive sample $\left({ }^{88} \mathrm{Zr}, \mathrm{t}_{1 / 2}=83.4\right.$ days), which was recently reported to have an extremely large thermal neutron capture cross section and resonance integral [1, 2, are planned for the winter of 2021. A performance overview, brief details on the ${ }^{88} \mathrm{Zr}$ fabrication and proof of good operation results will be presented.
\end{abstract}

\section{Introduction}

The accurate quantification of radiative neutron capture cross sections is essential in various applications such as radiochemical diagnostics, nuclear forensics and nuclear astrophysics. Although several studies have been performed on stable nuclei and a few on long-lived radionuclides [3] using direct techniques, the measurement of $(n, \gamma)$ cross sections on short-lived radionuclides is far more challenging due to backgrounds from the decay.

Hence, a number of indirect methods have been pursued and substantial effort has been devoted to quantify systematic errors associated with these techniques. The most popular indirect techniques include the surrogate method [4], the $\gamma$-ray strength function method [5, 6, the Oslo method [7] and the $\beta$-Oslo method [8].

Total cross section measurements are less affected by the decay background due to the long sample-to-detector distances, which are typically of the order of tens of meters. From neutron transmission measurements, $(n, \gamma)$ cross sections can be tightly constrained and even accurately calculated through the Nuclear Statistical Model (NSM) [9].

\footnotetext{
*e-mail: athanasios.stamatopoulos@lanl.gov
} 


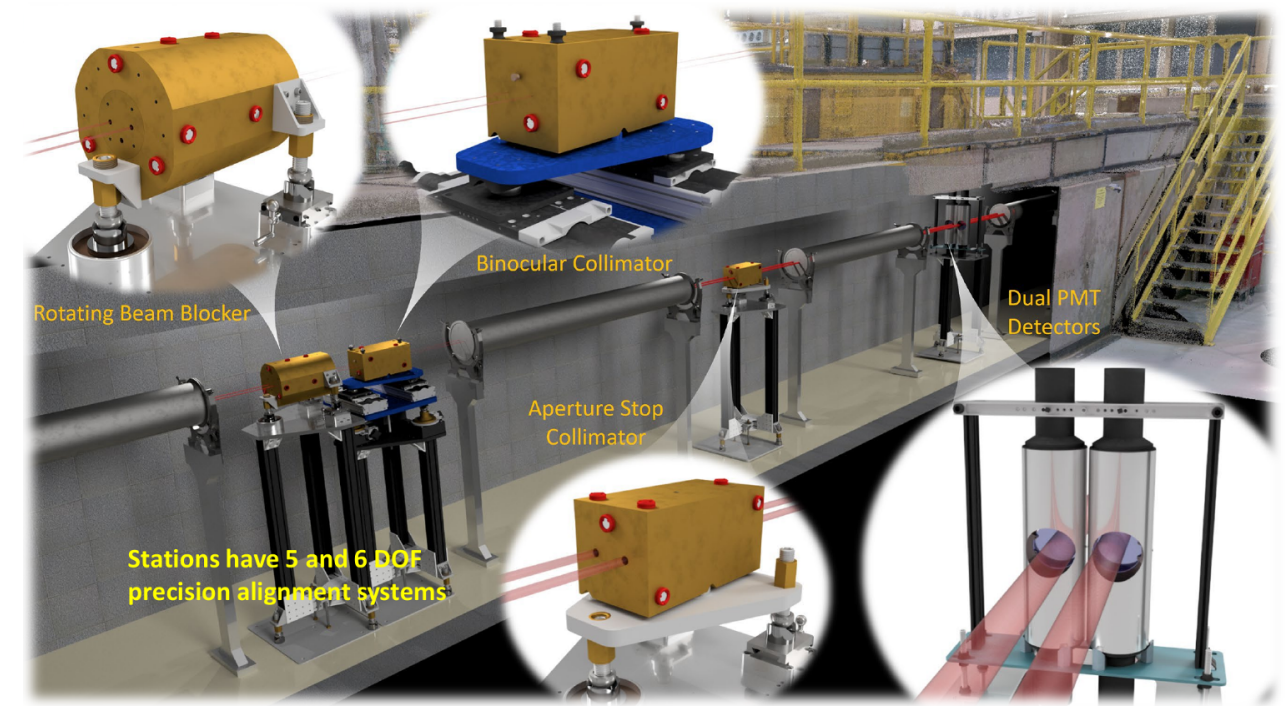

Figure 1. The DICER instrument is located at flight path 13, at Manuel Lujan Jr. Neutron Scattering Center at LANSCE. The collimation components along with the $31 \mathrm{~m}$ detection station are shown.

The technique is presented in detail in Ref. [10]. Briefly, neutron transmission spectra provide directly the level spacing $\left(D_{0}\right.$, distance between transmission dips), the total $\left(\Gamma\right.$, width of the transmission dip) and neutron resonance widths $\left(\Gamma_{n}\right.$, depth of the transmission dip). There are resonances where the radiation width $\left(\Gamma_{\gamma}\right)$ is not significantly smaller than $\Gamma_{n}$ and at the same time other reaction channels are suppressed. In those cases, the $\Gamma_{\gamma}$ can be easily calculated: $\Gamma=\Gamma_{n}+\Gamma_{\gamma} \cdot \Gamma_{\gamma}$ distributions are known to have small fluctuations, therefore only a small number of resonances is essentially needed to calculate the average radiation width $\left(\left\langle\Gamma_{\gamma}\right\rangle\right)$ with a sufficient accuracy.

The design of the Device for Indirect Capture Experiments on Radionuclides (DICER) [9, 11, 12], was based on the aforementioned technique and is being developed at the Los Alamos Neutron Science Center (LANSCE) where a high neutron flux is delivered. A plethora of radionuclides relevant to radiochemical diagnostics, nuclear forensics, nuclear astrophysics and nuclear data in general [9, 11, 13, are expected to be studied at DICER. The instrument is designed to study small samples (tens of $\mu \mathrm{g}, 0.1-1 \mathrm{~mm}$ in diameter) with half-lives of the order of tens of days or higher and level spacings of the order of tens of $\mathrm{eV}$ or smaller.

\section{The DICER instrument}

DICER is located at the Manuel Lujan Jr. Neutron Scattering Center (flight path 13) at LANSCE. Source-to-detector distances of 31 and a $64 \mathrm{~m}$ are available, however, only the $31 \mathrm{~m}$ station, shown in Fig. 1, will be discussed. A detailed description of the first DICER generation is provided in Ref. [14]. 


\subsection{Neutron source}

The neutron beam delivered at DICER is produced through spallation from LANSCE's $800 \mathrm{MeV}$ proton beam, pulsed at $20 \mathrm{~Hz}[15]$. The beam is impinging on a split, $10 \mathrm{~cm}$ in diameter, cylindrical tungsten target. Each proton pulse has a $125 \mathrm{~ns}$ FWHM and average intensity of $\sim 100 \mu \mathrm{A}$. The spallation neutrons are moderated in a liquid hydrogen moderator, a process which results in a neutron spectrum that spans from meV-MeV in energy regime, as shown in Fig. 2.

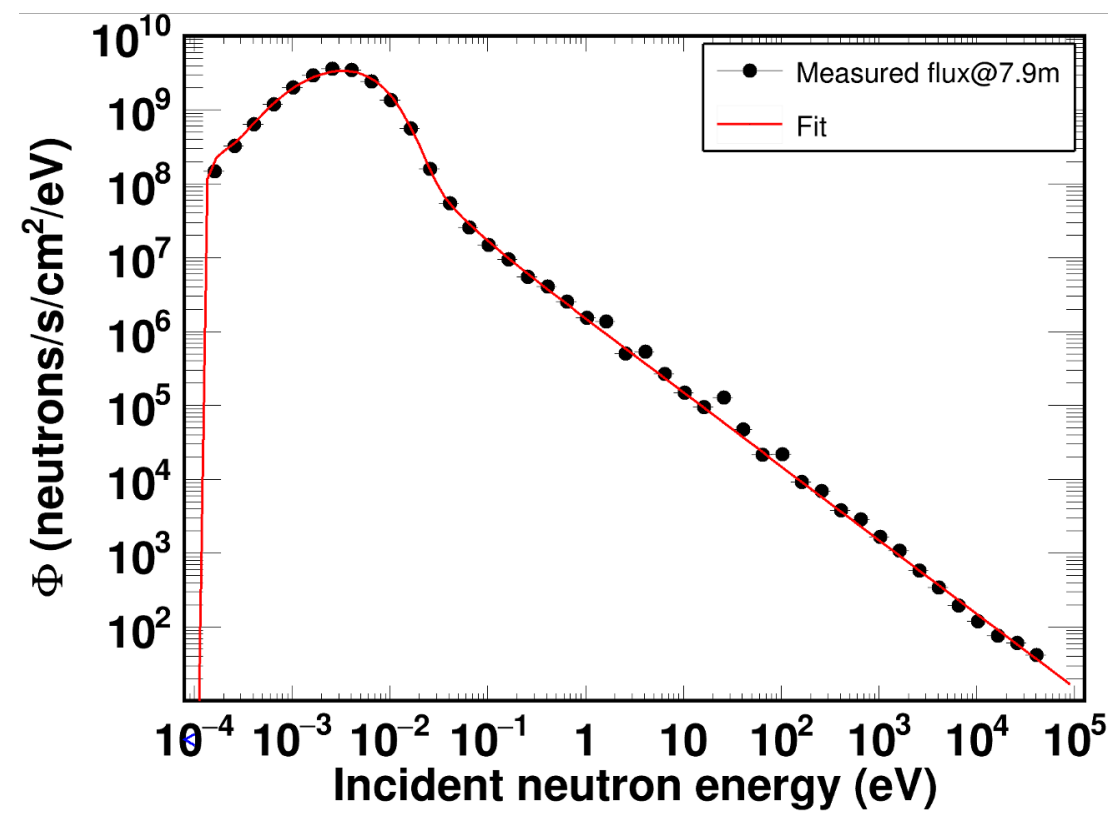

Figure 2. Measured and analytical description of DICER's neutron spectrum at $7.9 \mathrm{~m}$ from the spallation target.

\subsection{Collimation system}

The collimation system allows DICER to perform simultaneously sample in and sample out measurements, unlike the traditional neutron transmission measurements where periodic sample insertion/removal is required. Periodically inserting and removing the sample from the beam, involves positioning errors, therefore the samples used in traditional measurements are significantly larger than the beam size. In summary, the DICER approach allows the measurement of small samples and reduces the measuring time by a factor of two by utilising two non-parallel neutron beam lines, which converge at the same spot on the liquid hydrogen moderator. A brief description on how this is achieved, will be provided below.

A cylindrical shell of brass (Rotating Beam Blocker, Fig. 1), $30.5 \mathrm{~cm}$ in length and $21.5 \mathrm{~cm}$ in diameter, is installed at a $14.35 \mathrm{~m}$ distance from the spallation target. A rotating cylindrical piece of brass, $10 \mathrm{~cm}$ in diameter, is inserted in the cylindrical shell and is equipped with three $0.8 \mathrm{~cm}$ diameter holes, which provide four different beam line configurations: (a) two beam lines, (b) no beam lines, (c) a beam line to the left and (d) a beam line to the right. 
At a $14.85 \mathrm{~m}$ distance from the exit of the neutron source, a right rectangular prism made of brass (Binocular Collimator, Fig. 1), $30 \mathrm{~cm}$ long and $15 \mathrm{~cm}$ wide, is installed. This component serves both as a collimator and sample holder and provides two well defined and narrow beam lines, $1 \mathrm{~mm}$ in diameter, which point to the same area on the moderator, as illustrated in Fig. 3. The binocular collimator can house a cylindrical sample envelope of $1.5 \mathrm{~cm}$ in length and $1 \mathrm{~cm}$ in diameter. The positioning of the sample canisters with respect to the binocular collimator are pictured in Fig. 3 .

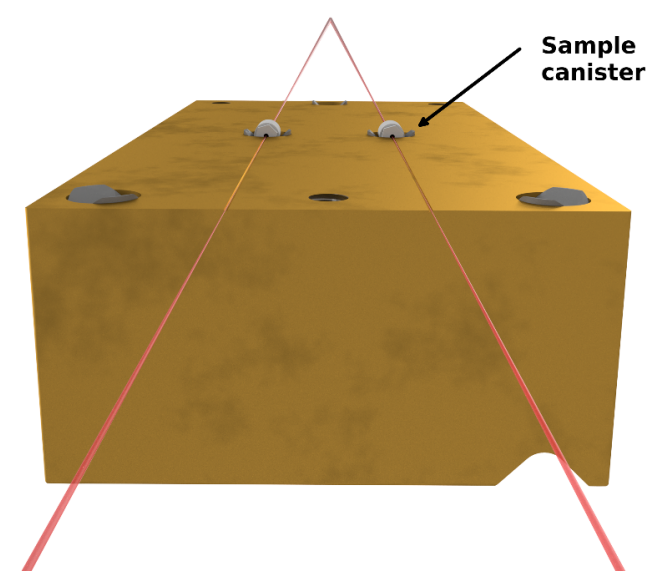

Figure 3. The binocular collimator of DICER allows to perform simultaneous sample in and out measurements, while it also serves as a sample holder.

Finally, the last shaping of the neutron beam takes place at $18.5 \mathrm{~m}$ from the neutron moderator. At that position, a rectangle brass collimator (aperture stop, Fig. 1) $30 \mathrm{~cm}$ long and 15 wide, is located. The aperture stop cleans up the beam penumbra from the sample collimator and ensures two well defined beam spots at the detector position.

\subsection{Detectors}

The standard detection system of DICER consists of two ${ }^{6} \mathrm{Li}$-glass disks, $10 \mathrm{~cm}$ in diameter and various thicknesses $(1,2,4,6.3,12.7 \mathrm{~mm})$ coupled to dual photomultipliers (PMTs). The PMTs are perpendicular with respect to the neutron beam propagation, hence not interacting with neutrons. This helps minimizing backgrounds. The two detectors are installed $31 \mathrm{~m}$ from the neutron source and are shown in Fig. 1 .

\section{Production of radioactive material at LANSCE and neutronic considerations: The ${ }^{88} \mathrm{Zr}$ case}

The DICER approach relies on the synergy between two LANSCE facilities: the Manuel Lujan Jr. neutron scattering center and the Isotope Production Facility (IPF) [16, 17]. A demonstration of this collaboration is the production of the ${ }^{88} \mathrm{Zr}$ radionuclide. Briefly, $29 \mathrm{~g}$ of a cylindrical yttrium metallic target, $2.90 \mathrm{~mm}$ in length 


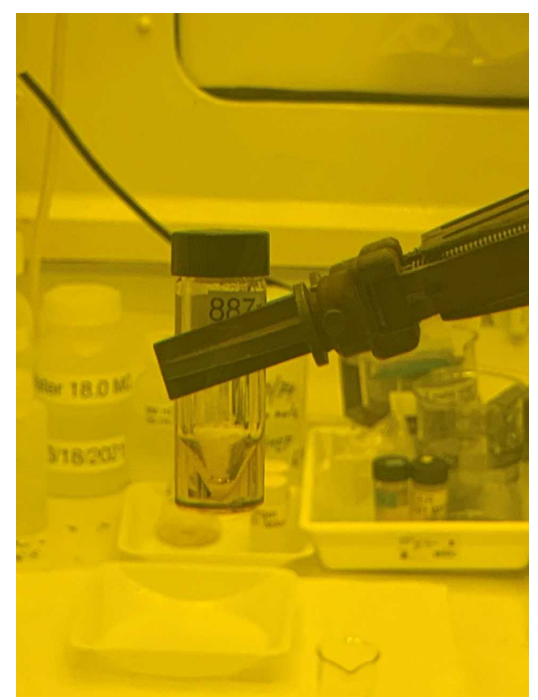

Figure 4. About $200 \mathrm{mCi}$ of ${ }^{88} \mathrm{Zr}$ were produced at IPF at LANSCE. The radioactive material is diluted in $1.9 \mathrm{~mol} / \mathrm{L} \mathrm{DCl}$. The image was taken inside a hot cell.

and $46 \mathrm{~mm}$ in diameter, encapsulated in an aluminum holder, was irradiated at the IPF.

The target was irradiated for a total of $\sim 9$ hours at an average beam current of $96.1 \mu \mathrm{A}$. After cooling the irradiated material for 82 days, the target was disassembled and dissolved in a hot cell environment in $150 \mathrm{~mL}$ of $6 \mathrm{~mol} / \mathrm{L} \mathrm{HCl}$ by slow addition of $5-10 \mathrm{~mL}$ portions. About $200 \mathrm{mCi} / 8 \mathrm{GBq}$ of ${ }^{88} \mathrm{Zr}$ were separated and diluted in $1.9 \mathrm{~mol} / \mathrm{L} \mathrm{DCl}$ solution. Finally, the solution was evaporated almost to dryness and washed with $10 \mathrm{~mL}$ of $6 \mathrm{~mol} / \mathrm{L} \mathrm{HCl}$. This solution was transferred into a $10 \mathrm{~mL}$ v-vial. The sample is shown in Fig. 4.

The successful yttrium irradiation and ${ }^{88} \mathrm{Zr}$ recovery was confirmed through the means of gamma-ray spectroscopy, using a High Purity Germanium (HPGe) detector and a small aliquot $(25 \mu \mathrm{L})$ of the separated material. The aliquot was diluted in 10 $\mathrm{mL}$ of $1 \mathrm{~mol} / \mathrm{L} \mathrm{HCl}$ and was then placed in front of an HPGe detector. The recorded spectrum shown in Fig. 5, illustrates the successful production and separation of ${ }^{88} \mathrm{Zr}$ : The $392.7 \mathrm{keV}$ photo-peak from ${ }^{88} \mathrm{Zr}$ and the 898.7 and $1836.8 \mathrm{keV}$ from the ${ }^{88} \mathrm{Y}$ daughter are dominating the spectrum.

Since DICER is an instrument that measures all neutron reactions that take place during a measurement, it has to be ensured that the chemical format/solution of the sample is fairly transparent to neutrons. In other words, the minimum amount of neutron interactions with materials other than the one of interest ${ }^{88} \mathrm{Zr}$ in this example) is preferred. Hydrogen has a significant high probability to scatter neutrons, therefore is appreciably not transparent to neutrons. To mitigate that effect, $\mathrm{HCl}$ was replaced with $\mathrm{DCl}$. The ${ }^{88} \mathrm{Zr}+\mathrm{HCl}$ sample was dried once again and the same procedure described above was used to produce ${ }^{88} \mathrm{Zr}$ in DCl solution. As shown in Fig. 6. the transmission through $\mathrm{DCl}$, for a $1.4 \mathrm{~mol} / \mathrm{L}, 1 \mathrm{~cm}$ long and $1.2 \mathrm{~mm}$ in diameter cylindrical sample, ranges between $85-95 \%$ in the region where a ${ }^{88} \mathrm{Zr}$ resonance is expected. For this calculation, (n,tot) cross sections from the ENDF/B-VIII.0 library [18] were used for ${ }^{2} \mathrm{H},{ }^{35} \mathrm{Cl}$ and ${ }^{37} \mathrm{Cl}$. 


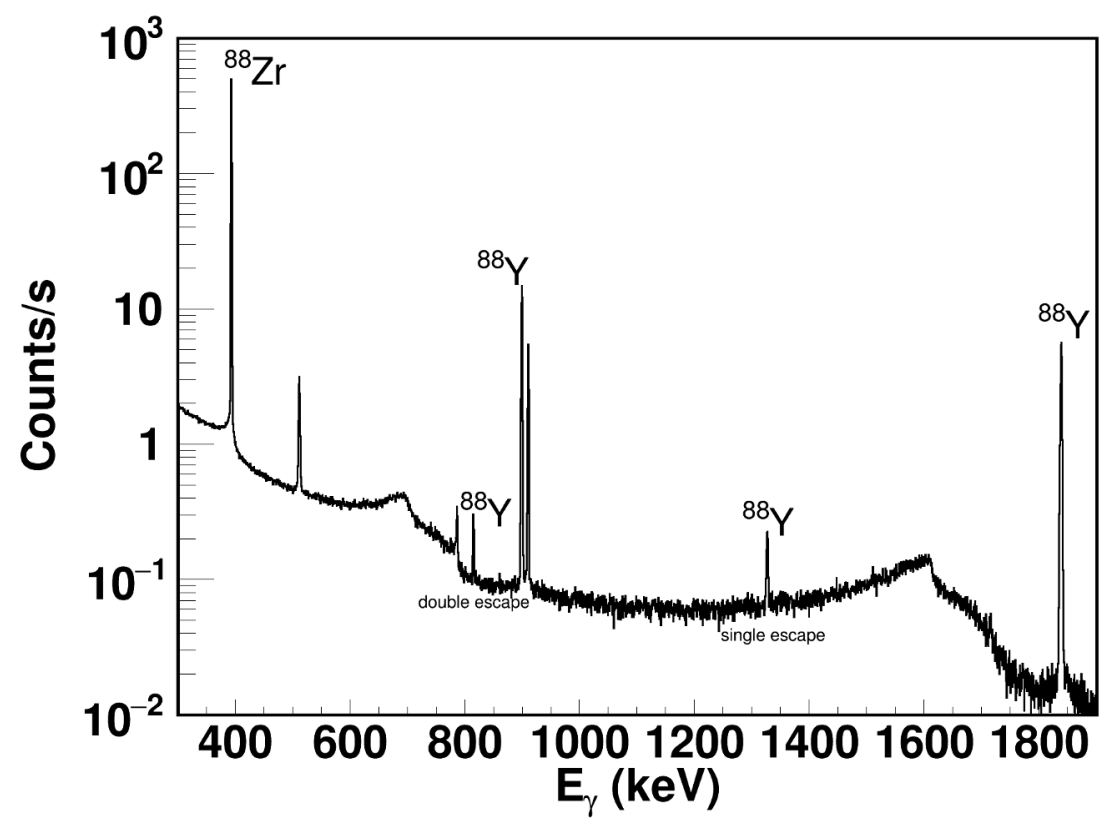

Figure 5. Gamma ray spectrum of $25 \mu \mathrm{L}$ of the sample taken with an HPGe detector. Only the ${ }^{88} \mathrm{Zr}$ and ${ }^{88} \mathrm{Y}$ gamma rays along with their escape peaks are labeled.

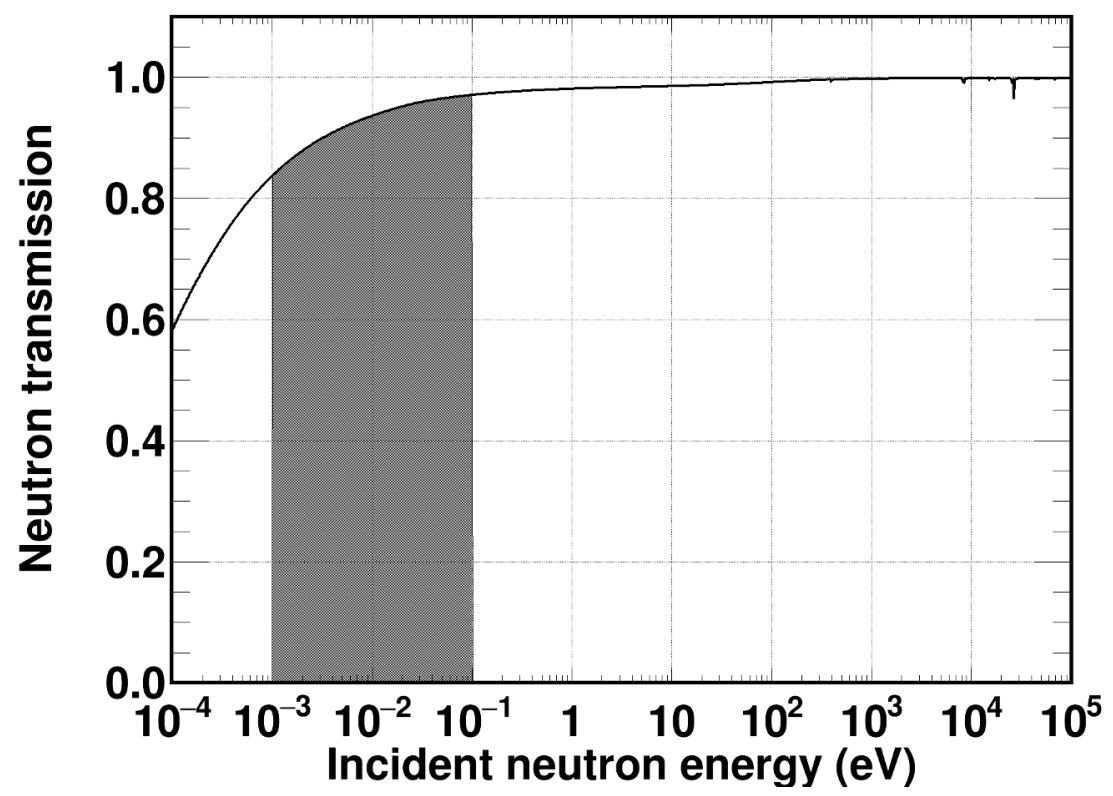

Figure 6. Neutron transmission through a $1.4 \mathrm{~mol} / \mathrm{L}, 1 \mathrm{~cm}$ long and $1.2 \mathrm{~mm}$ in diameter cylindrical sample made of DCl. The grey shaded area corresponds to the main area of interest where a ${ }^{88} \mathrm{Zr}$ resonance is expected. ENDF/B-VIII.0 cross sections for ${ }^{2} \mathrm{H},{ }^{35} \mathrm{Cl}$ and ${ }^{37} \mathrm{Cl}$ were used. 


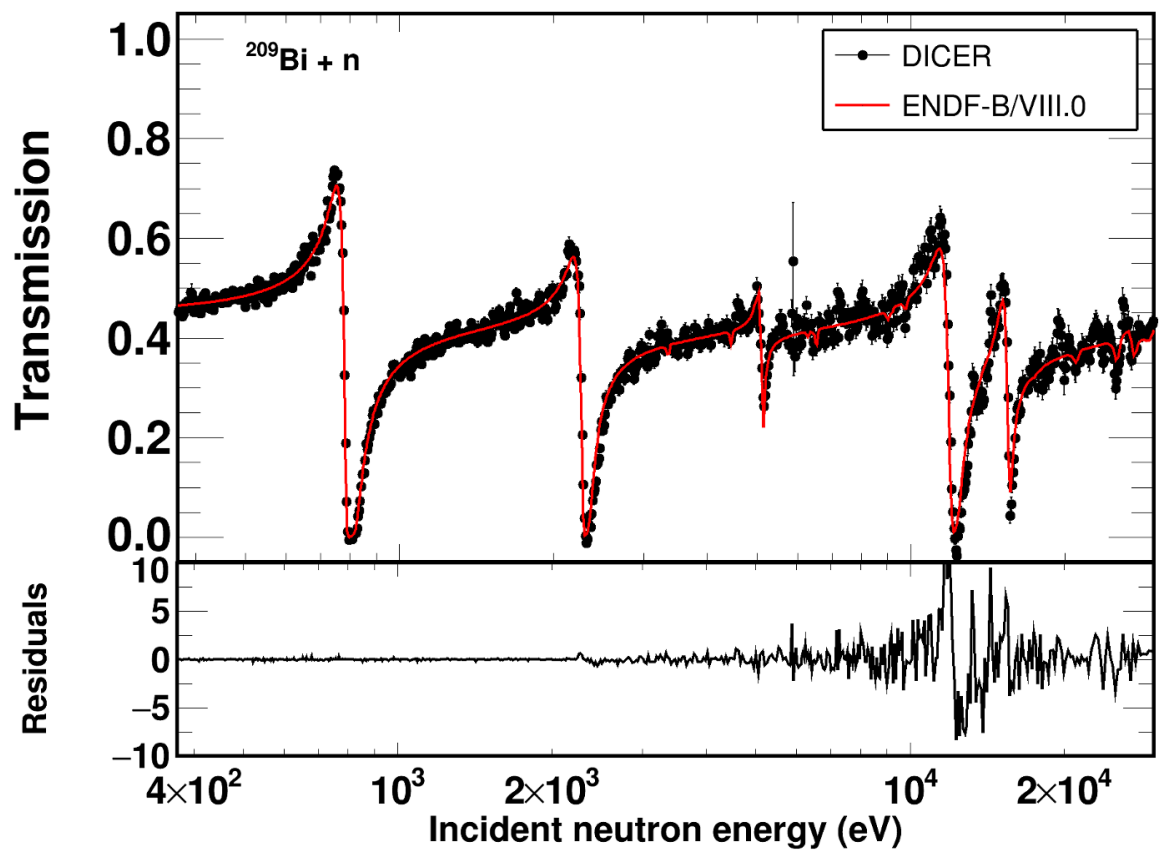

Figure 7. DICER data in comparison with ENDF/B-VIII.0. A satisfactory reproduction was achieved.

\section{Performance overview}

DICER is being commissioned since the autumn of 2019 and although the commissioning phase will finish at the end of 2022, many stable nuclei have already been studied such as ${ }^{147,149} \mathrm{Sm},{ }^{191,193} \mathrm{Ir},{ }^{95} \mathrm{Mo},{ }^{209} \mathrm{Bi},{ }^{197} \mathrm{Au}$, ${ }^{\text {nat }} \mathrm{Cd}$ and ${ }^{\text {nat }} \mathrm{Gd}$. To demonstrate the good understanding of the new device and the reproduction of well-known reactions, Fig. 7 shows a satisfactory reproduction of DICER data using ENDF/B-VIII.0 resonance parameters.

\section{Conclusion}

A new instrument at flight path 13 at the Manuel Lujan Jr. Neutron Scattering Center of LANSCE is being developed to study indirect neutron capture on short-lived radionuclides, through neutron transmission measurements and resonance analysis. The DICER concept is based on the synergy between experimental facilities at LANSCE, such as production of radioactive samples at IPF and their neutron irradiation at the Manuel Lujan Jr. Neutron Scattering Center. Great effort has been made to design and precisely align the instrument as well as to develop an efficient and reliable data reduction scheme. Both efforts led to a deep understanding of the instrument's performance, which was demonstrated by the satisfactory reproduction of DICER data from evaluated resonance parameters [14]. Finally, DICER is getting ready to perform its first measurements on radioactive samples on ${ }^{88} \mathrm{Zr}$ and ${ }^{88} \mathrm{Y}$. 


\section{Acknowledgements}

This work benefited from the use of the LANSCE accelerator facility and was supported by the US Department of Energy through the Los Alamos National Laboratory. Los Alamos National Laboratory is operated by Triad National Security, LLC, for the National Nuclear Security Administration of US Department of Energy (Contract No. 89233218CNA000001). Research presented in this article was supported by the Laboratory Directed Research and Development program of Los Alamos National Laboratory under project number 20200108DR.

\section{References}

[1] J.A. Shusterman, N.D. Scielzo, K.J. Thomas, E.B. Norman, S.E. Lapi, C.S. Loveless, N.J. Peters, J.D. Robertson, D.A. Shaughnessy, A.P. Tonchev, Nature 565, 328 (2019)

[2] J.A. Shusterman, N.D. Scielzo, E.P. Abel, H.K. Clause, N.D. Dronchi, W.D. Frey, N. Gharibyan, J.A. Hart, C.S. Loveless, S.R. McGuinness et al., Phys. Rev. C 103, 024614 (2021)

[3] A. Couture, R. Reifarth, At. Data and Nucl. Data Tables 93, 807 (2007)

[4] J.E. Escher, J.T. Burke, R.O. Hughes, N.D. Scielzo, R.J. Casperson, S. Ota, H.I. Park, A. Saastamoinen, T.J. Ross, Phys. Rev. Lett. 121, 052501 (2018)

[5] H. Utsunomiya, S. Goriely, H. Akimune, H. Harada, F. Kitatani, S. Goko, H. Toyokawa, K. Yamada, T. Kondo, O. Itoh et al., Phys. Rev. C 82, 064610 (2010)

[6] H. Utsunomiya, S. Goriely, T. Kondo, C. Iwamoto, H. Akimune, T. Yamagata, H. Toyokawa, H. Harada, F. Kitatani, Y.W. Lui et al., Phys. Rev. C 88, 015805 (2013)

[7] M. Guttormsen, T. Ramsøy, J. Rekstad, Nucl. Instrum. Meth. A 255, 518 (1987)

[8] A. Spyrou, S.N. Liddick, A.C. Larsen, M. Guttormsen, K. Cooper, A.C. Dombos, D.J. Morrissey, F. Naqvi, G. Perdikakis, S.J. Quinn et al., Phys. Rev. Lett. 113, 232502 (2014)

[9] P. Koehler, Tech. Rep. LA-UR-14-21466, Los Alamos National Laboratory (2014), https://permalink.lanl.gov/object/tr?what=info: lanl-repo/lareport/LA-UR-14-21466

[10] P.E. Koehler, K.H. Guber, Phys. Rev. C 88, 035802 (2013)

[11] P. Koehler, et al., Tech. Rep. LA-UR-14-21656, Los Alamos National Laboratory (2014), https://permalink.lanl.gov/object/tr?what= info:lanl-repo/lareport/LA-UR-14-21656

[12] P. Koehler, et al., Tech. Rep. LA-UR-18-22995, Los Alamos National Laboratory (2018)

[13] G. Keksis, et al., Tech. Rep. LA-UR-21-23034, Los Alamos National Laboratory (2021), DRAFT

[14] A. Stamatopoulos, P. Koehler, A. Couture, B. DiGiovine, G. Rusev, J. Ullmann, Nucl. Instrum. Meth. A 1025 (2022)

[15] P.W. Lisowski, K.F. Schoenberg, Nucl. Instrum. Meth. A 562, 910 (2006)

[16] K.F. Johnson, H.W. Alvestad, W.C. Barkley, D.B. Barlow, D.S. Barr, L.S. Bennett, L.J. Bitteker, E.A. Bjorklund, W. Boedeker, M.J. Borden et al. (2004)

[17] LANSCE, Ipf, https://lansce.lanl.gov/facilities/ipf/index.php

[18] D. Brown, M. Chadwick, R. Capote, A. Kahler, A. Trkov, M. Herman, A. Sonzogni, Y. Danon, A. Carlson, M. Dunn et al., Nucl. Data Sheets 148, 1 (2018) 